\title{
Applicability of a Hall Sensor in Directional Ground Fault Protections of MV Cable Networks with No-Effective Earthing
}

\author{
Marcin Habrych ${ }^{1}$, Bogdan Miedzinski ${ }^{2}$, Xin Wang ${ }^{3}$ \\ ${ }^{1}$ Department of Electrical Engineering, Wroclaw University of Technology, \\ Wybrzeze Wyspianskiego 27, 50-370 Wroclaw, Poland \\ ${ }^{2}$ Institute of Innovative Technologies, EMAG, \\ Leopolda 31, 40-189 Katowice, Poland \\ ${ }^{3}$ China Agricultural University, College of Engineering, \\ Qinghua Donglu 17, Haidian District Beijing 100083, China \\ marcin.habrych@pwr.edu.pl
}

\begin{abstract}
${ }^{1}$ Abstract-Paper presents and discusses results of analysis and research on the possibility of use of the Hall sensor as measuring element in directional ground fault protection. The considerations have been carried out for the new balance transformer structure with magnetic shielding. Its applicability has been confirmed by tests under real ground faults in MV networks of $6 \mathrm{kV}$ and $20 \mathrm{kV}$ with no-effective earthing. On the basis of the investigated results appropriate practical conclusions have been formulated.
\end{abstract}

Index Terms-Directional protection; ground fault; Hall sensor; MV cable network; power system protection.

\section{INTRODUCTION}

One of the basic requirements for electric protections (regardless of application) is respective sensitivity of operation while maintaining its selectivity. In the case of ground fault protections means that they should be able to detect and correctly localize all possible earth faults in practice including these of a small current value (below $1 \mathrm{~A}$ ) under resistive short-circuits in isolated networks [1]-[3]. Such situation exists, inter alia, in mining cable MV networks with no-effective earthing in which problems with detection and selective clearing of single phase ground faults by electrical protection of relatively low sensitivity using the conventional residual current transformer systems are well known [4]-[10].

The possibility of sensitive adjusting of the ground fault protections is seen in the use of appropriate types of current transmitters in this Hall sensors [11], [12]. Previous work carried out in this field by the authors show that there is a real possibility to construct a sensitive current transmitter, designed especially for directional earth fault protections of MV networks provided optimum magnetic core structure adequately adapted to the dimensions of power cables and cable lines working conditions [13], [14]. This paper presents a new structure of a directional ground fault protection of a MV networks with no-effective earthing basing on use of a Hall as a measuring device. The results of

Manuscript received 12 April, 2016; accepted 11 August, 2016. theoretical analysis, laboratory tests on physical models and testing in real conditions under 1-phase earth faults in MV cable networks of an opencast mine has been discussed.

\section{General CONCEPT OF THE Protection}

Way to solve the directional ground fault protection based on the Hall sensor is shown in Fig. 1.

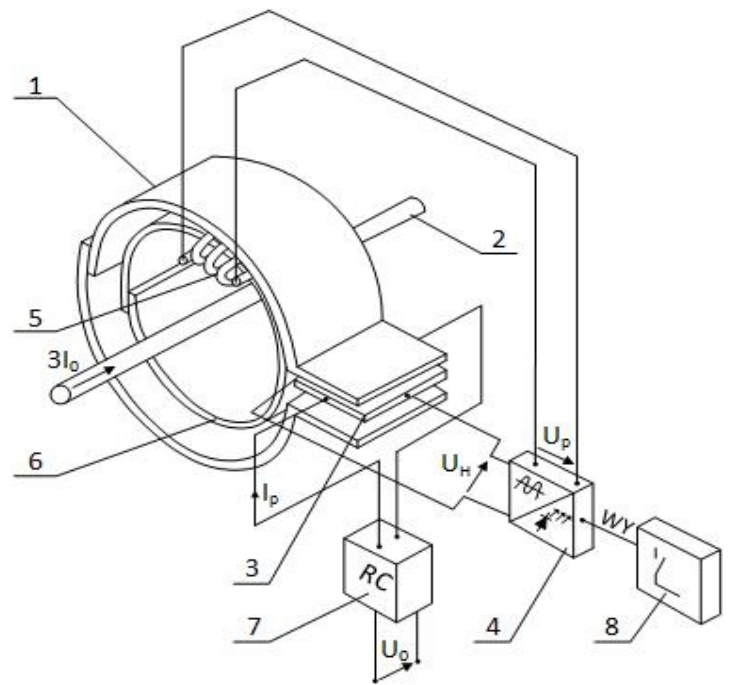

Fig. 1. Simplified scheme of the directional ground fault protection with a Hall sensor as a detector; 1 - core balance CTs, 2 - 3-phase cable, 3 - Hall sensor, 4 - measuring unit with fibre optic output, 5 - auxiliary winding $\left(z_{p}\right), 6$ - magnetic screen, 7 - phase shifter, 8 - output element.

It consists of a zero sequence current transformer made from several magnetic sheet metal coil (SURA - type M-6) and applied directly to the power cable of the protected feeder. Inside the transformer's window is located a magnetic shield made of the same cold-rolled magnetic steel (1-2 turns). The Hall sensor is situated in the specially shaped air gap of the transformer core and measure the value of magnetic flux density (flux $\phi_{0}$ ) produced by zero sequence current $\left(3 I_{0}\right)$ equivalent to the earth fault current. The electric field however, affecting the Hall sensor is produced by the zero sequence voltage $\left(U_{0}\right)$ fed from the open delta voltage 3-phase transformers system. An 
additional coil is wound on the magnetic screen (of $z_{0}$ number of turns) to provide autonomous power to an output unit of the protection. The principle of operation of the directional protection is based on the fact that for sinusoidal waveform of both the ground fault current $\left(3 I_{0}\right)$ and zero sequence voltage $\left(U_{0}\right)$, a magnetic flux $\left(\phi_{0}(t)\right)$ and therefore, the magnetic field intensity in the gap $\left(H_{0}(t)\right)$ (under the influence of which is the Hall sensor), the bias current $\left(I_{p}(t)\right)$ and the Hall voltage $\left(U_{H}(t)\right)$ can be expressed by the following relations:

$$
\begin{gathered}
\varphi_{0}(t)=k_{1}\left(3 I_{0 m}\right) \sin \left(\omega t+\phi_{0}\right), \\
I_{p}(t)=k_{2} U_{0 m} \sin \omega t, \\
U_{H}(t)=k_{3} \phi_{0}(t) I_{p}(t),
\end{gathered}
$$

where $3 I_{0 m}, U_{0 m}$ - amplitude of zero sequence current and voltage respectively, $k_{1}, k_{2}, k_{3}$ - factors dependent on the parameters of current zero-sequence filter, voltage filter and the Hall sensor respectively [11], $\varphi_{0}-$ the phase angle between $\left(3 I_{0}\right)$ and $\left(U_{0}\right), \omega$ - angular frequency.

After the introduction of additional internal angle $(\psi)$ and respective transformation we obtain

$$
\begin{gathered}
U_{H}(t)=k_{3} U_{0 m}\left(3 I_{0 m}\right) \times \\
\times\left[\cos \left(\phi_{0}-\psi\right)-\cos \left(2 \omega t+\phi_{0}-\psi\right)\right],
\end{gathered}
$$

where $\psi$ - internal angle (called a relay characteristic angle (RCA)) adjusted as required by means of a phase shifter.

Considering above it is seen that the output Hall voltage has two components: DC components $\left(U_{H D C}\right)$ dependent on the phase shift $\varphi_{0}$ value and variable component $\left(U_{H A C}\right)$ of a double frequency $(100 \mathrm{~Hz})$

$$
U_{H}(t)=U_{H D C}(t)+U_{H A C}(t) .
$$

The DC component may therefore, be used successfully as a criterion for directional protection operation. However, in order to ensure its maximum value (for networks with different no-effective earthing) it is necessary to make an appropriate angle correction (RCA) using the phase shifter circuit further arranged into the Hall sensor polarization system (for example in isolated networks the value of the phase angle $\varphi_{0} \approx 90^{\circ}$ ) [2], [13]. Due to the fact that the determination of the angle value $\left(\cos \varphi_{0}\right)$ is associated with the consideration based on the measurement results obtained from the maximum values of current and voltage (so as to eliminate this inconvenience), it is proposed to use a measuring algorithm as shown in Fig. 2.

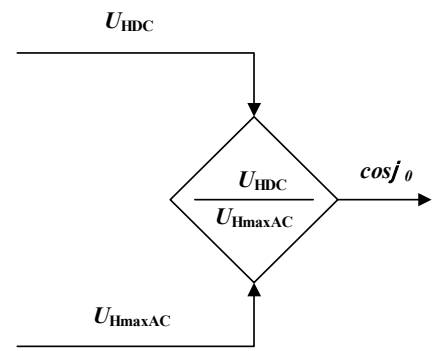

Fig. 2. Algorithm executing the function of angle $\left(\cos \varphi_{0}\right)$ measurement.
It should be noted, that the voltage $U_{H}(t)$ waveform according to (4) is true only for the full symmetry of the parameters of the used Hall sensor. The Hall sensor however, usually displays some parameter's asymmetry, what in turn may affect the measurement accuracy of the phase angle [14], [15]. Measurement of the phase angle is made under different magnetic field polarity so as bias current and hence there might be some differences in the Hall voltage transient waveforms resulting from the asymmetric effects of both current and voltage of the sensor [4], [5], [12], [15].

Studies have shown that changes in the amplitude of the instantaneous values of $U_{H A C}$ mainly run but do not interfere with the angular relationship $\varphi_{0}$ (Fig. 3).

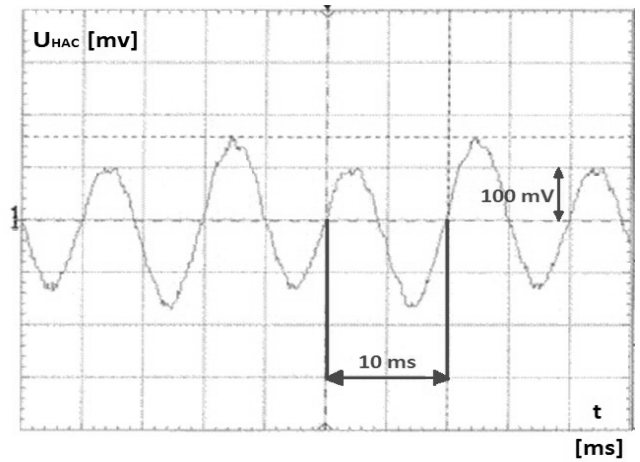

Fig. 3. An example of the variable component $U_{H A C}(t)$ for sinusoidal magnetic field values so as bias current.

The observed asymmetry of the Hall sensor parameters on the value of the DC component $U_{H D C}$ is also greatly reduced compared to the variable $U_{H A C}$.

\section{Sensitivity PRoblems OF The Protection}

Sensitivity of the ground fault protection depends primarily on the value of a zero sequence current $\left(3 I_{0}\right)$ and hence the value of magnetic flux density $B$ generated by this short-circuit current in the gap of the core (in which is arranged the Hall sensor). This is obviously associated with both the magnetic properties of the material and the geometric dimensions of the core and air gap. For practical reasons, about how to resolve the structural core of the transformer to allow its direct location on the cable (without disconnecting the cable from power supply) as the magnetic material used a cold-rolled sheet (SURA) of a single strip width of $15 \mathrm{~mm}$ and a thickness of $0.25 \mathrm{~mm}$ respectively. Average magnetization curve of the material is shown in Fig. 4.

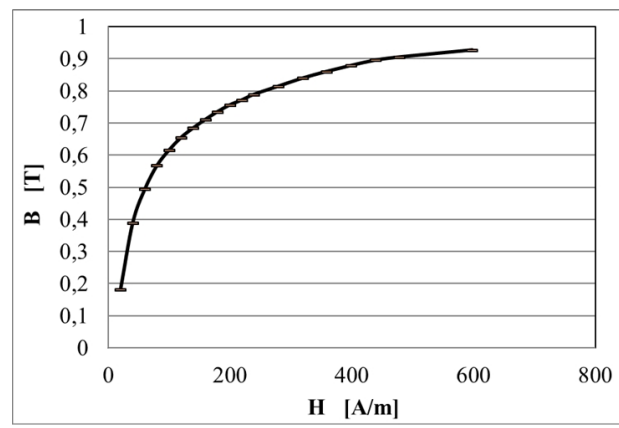

Fig. 4. Medium $B-H$ curve of SURA magnetic material.

Simulation analysis of the distribution and values of the 
magnetic field for different operation conditions of the Halleffect transducer carried out by means of computer program and visualization, concerned with the effects, by application of "Magnetic-Nodal" option of the program. An exemplary distribution of magnetic flux lines and the magnetic induction in the transformer for the fault current $3 I_{0}=2 \mathrm{~A}$ are shown in Fig. 5 and Fig. 6 respectively. To verify the effectiveness before the tests in real conditions respective laboratory investigations simulating the operation of the protection under various faults were carried out (enforced by appropriate waveforms and values as ground fault current and zero sequence voltage). However, prior to working numerous simulation study using EMTP were performed as well.

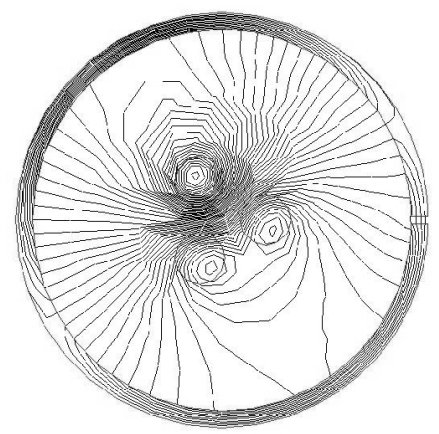

Fig. 5. Magnetic flux lines distribution (2D) in the transformer for $3 I_{0}=$ 2 A.

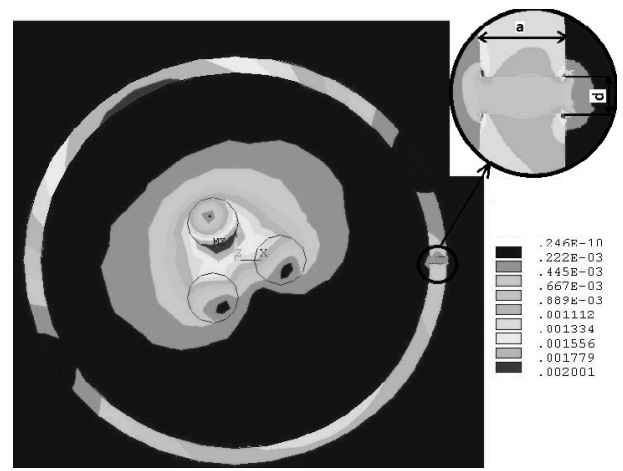

Fig. 6. Magnetic induction $(B)$ distribution (2D) in the transformer for $3 I_{0}=$ 2 A.

For the assumed constant cross-sectional area of the core equal to about: $S=60 \mathrm{~mm}^{2}$ and the average magnetization path: $l_{a}=(25-40) \mathrm{mm}$, conducted adequate analyses of changes in the value of induction in the air gap, depending on the width $(a)$ of the core and the length $(d)$ of the air gap (see $a, d$ in Fig. 6).

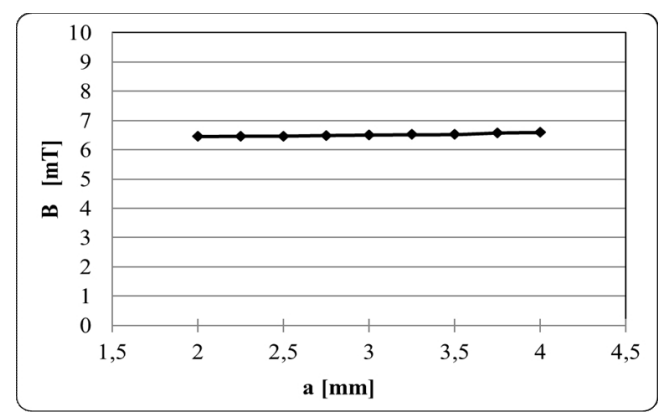

Fig. 7. Flux density $B$ inside the air gap versus width $(a)$ of the core for $3 I_{0}=50 \mathrm{~A}(d=1.8 \mathrm{~mm})$.

It has been found that for constant cross-sectional area of the core when changing its width as well as the average magnetization path (in the analysed range) it has not been found a major impact on the value of the induction $B$ in the air gap. The crucial factor is, of course, the length $(d)$ as for example shown in Fig. 7 and Fig. 8.

For accepted, in the physical model of the air gap dimensions (imposed upon the sensor), dependence of induction value as a function of the zero-sequence current $\left(3 I_{0}\right)$ exhibits linearity especially above $10 \mathrm{~A}$ (Fig. 9). This is due to the saturation of the transformer core material.

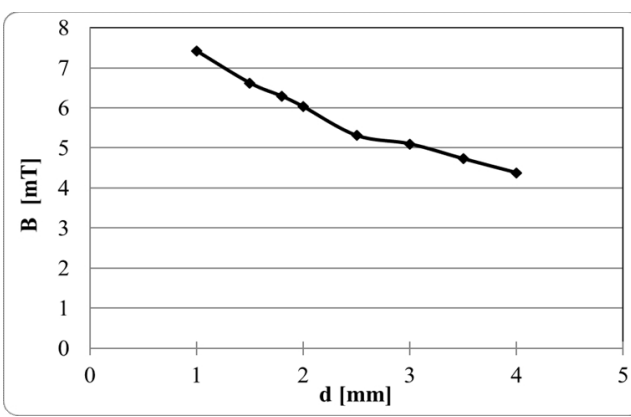

Fig. 8. Influence of the air gap length $(d)$ on flux density $B$ for $3 I_{0}=50 \mathrm{~A}$ $(a=15 \mathrm{~mm})$.

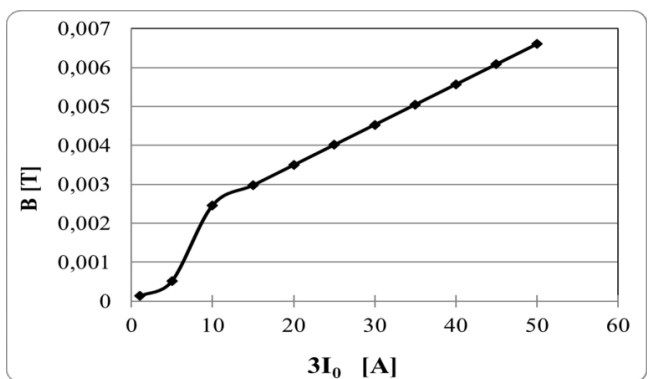

Fig. 9. Flux density within the gap area versus $3 I_{0}(a=15 \mathrm{~mm}, d=1 \mathrm{~mm})$.

\section{ShIELding OF THE 3-PHASE CABLE LOAD INTERFERENCE}

The main source of errors of Hall sensors is the impact of electromagnetic fields due to particular phase currents in the cable as well as interfering external field. For full symmetry of the 3-phase load in the Hall sensor output theoretically should not appear any signal since the resultant magnetic flux is equal to zero. However, given the fact that each phase of the 3-phase cable is positioned differently with respect to the magnetic gap hence, there is a certain asymmetry of electromagnetic circuits and related different values of the leakage flux for various phases (Fig. 10).

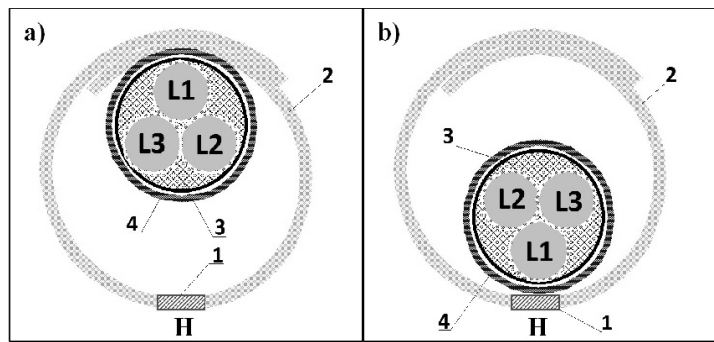

Fig. 10. Location of the 3 phase cable relating to the Hall sensor; 1-Hall sensor, 2-magnetic core of CTs, 3-power cable; 4-magnetic screen.

Consequently, even at full load current symmetry appears some error voltage at the output of the Hall sensor. Its value is the greater the higher the amplitude of the load current and the greater particular phase position asymmetry with 
respect to the air gap with the Hall sensor (Fig. 10(b)).

Studies have shown that reducing the interference of 3phase load current is obtained by applying the appropriate magnetic shielding placed directly on the cable (see Fig. 1). Such the screen made of several strips of the cold-rolled steel (SURA) is used in the developed directional ground fault protection based on the Hall sensor as a measuring unit. The appropriate simulations using computer program were carried out in order to determine the optimal structure and geometrical dimensions of the screen. Due to the structure symmetry only one half of the system could be mapped (as shown in Fig. 11). The guidance for the magnetic screen's dimensions has been formulated after confirmation by both laboratory and field tests for real physical device.

Studies performed for various thickness and length of the screen in relation to the core of the transformer in the range of changes in load current of the 3-phase cable from $1 \mathrm{~A}$ to 500 A [15] (exemplary results are shown in Fig. 12).

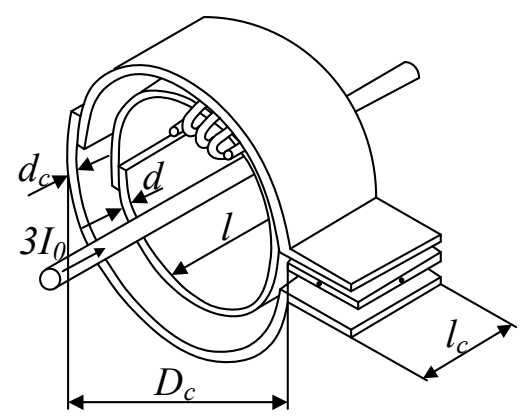

Fig. 11. Model of the protection for computations $\left(l, l_{c}-\right.$ length of the screen and transformer core; $d, d_{c}$ - thickness of the screen and the core respectively; $l_{c}=15 \mathrm{~mm}, d_{c}=3 \mathrm{~mm}, D_{c}$ - core diameter).

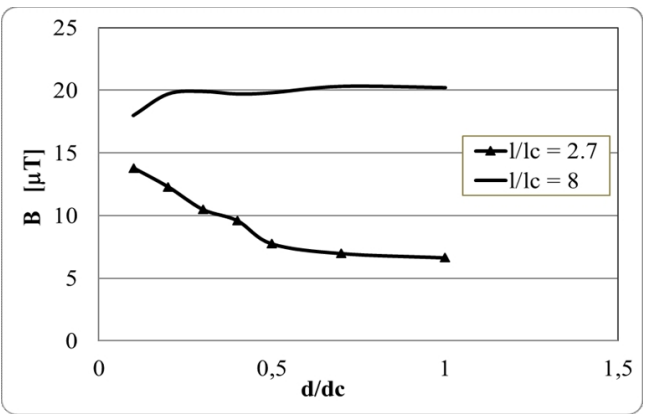

Fig. 12. Variation of the residual flux density, inside the air gap of the core due to the $10 \%$ load asymmetry $(\mathrm{L} 1=45 \mathrm{~A}, \mathrm{~L} 2=\mathrm{L} 3=50 \mathrm{~A})$, with the screen thickness $(d)\left(d_{c}=3 \mathrm{~mm}, l_{c}=15 \mathrm{~mm}\right)$.

It was found that the best results occur with the use of the screen width six times greater than the width of the transformer $\left(l / l_{c}=6\right)$ and the ratio of sectional area of the screen $\left(S_{E}\right)$ to the cross-sectional area of the transformer core $\left(S_{C}\right)$ equal to $S_{E} / S_{C}=1.125$. It should be noted that, although the use of the screen reduces the value of the magnetic field in the air gap however, ratio of the signal $(S)$ (during the ground fault) to noise $(N)$ (due to magnetic asymmetry of the 3-phase cable as well as external interference) is significantly (over $200 \%$ ) increased.

\section{PERformance of the Protection Under Ground FAULTS IN REAL MV NETWORKS WITH NO EFFECTIVE GROUNDING}

In order to verify the effectiveness of a ground fault protection using Hall sensor as a decisive unit the appropriate tests were performed in real mining networks of $20 \mathrm{kV}$ and $6 \mathrm{kV}$ with no effective grounding respectively as illustrated in Fig. 13.

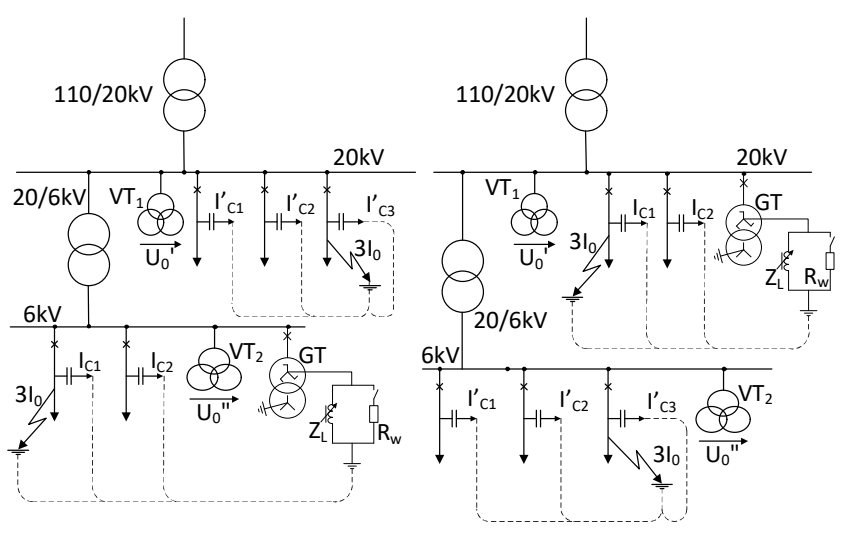

Fig. 13. A simplified equivalent circuit of MV mining networks (6 kV and $20 \mathrm{kV}$ ) for testing the efficiency of operation of the Hall sensor based ground fault protection (GT - grounding transformer, $z_{L}, R_{w}$ - impedance and resistance of the grounding system, VT1, VT2 - voltage transformer units with open delta connection).

Many years of experience of the authors on directional ground fault protections in MV networks have raised the idea of using for this purpose Hall sensor as a decisive making element. Conventional protections do not operate correctly under all ground fault's conditions particularly with regard to high resistance (over about $1.5 \mathrm{k} \Omega$ ) short circuit as shown by author's study. The main advantage of the proposed approach is primarily a much wider range of selective operation both under steady and transient states regardless of the structure simplicity, small size and price as well as ease of installation and service.

The networks under tests worked with or isolated neutral, compensated, using the Petersen coil $\left(z_{L}-\right.$ impedance included), as well as compensated together with an active current component injection (ACF system). In this last case the resistance $R_{w}$ is activated (to enforce the active component of about $20 \mathrm{~A}$ ) after approximately 3 seconds from the appearance of a zero sequence voltage $\left(U_{0}\right)$ equal to around $30 \mathrm{~V}$. The earth faults were carried out as permanent (phase connection to metallic housing of the substation), resistive (about $80 \Omega$ and over $2 \mathrm{k} \Omega$ fault resistance to the ground) and as a transient when the phase conductor fell to the ground. The MV networks were equipped with various units of ground fault protections commonly used like: delayed over-current, admittance and directional active or reactive power protection. To investigate the reliability and selectivity of operation of the Hall sensor based unit the examined protection was installed on both the healthy and faulty cable feeders. In isolated networks the relay characteristic angle (RCA) should be set at an angle equal to $90^{\circ}$ whereas, in these with compensation and/or grounded via resistor $R_{w}$ (ACF system) the RCA must be equal to $0^{\circ}$ respectively [1], [3], [11]. Under the tests both fault current $\left(3 I_{0}\right)$, zero-sequence component of voltage $\left(U_{0}\right)$ (residual voltage at open delta connection of 3 phase VTs unit) and value as well as polarity of the output constant component $\left(U_{H D C}\right)$ of the Hall sensor were controlled and recorded. The selected test results are 
provided in Fig. 14-Fig. 20 for example respectively.

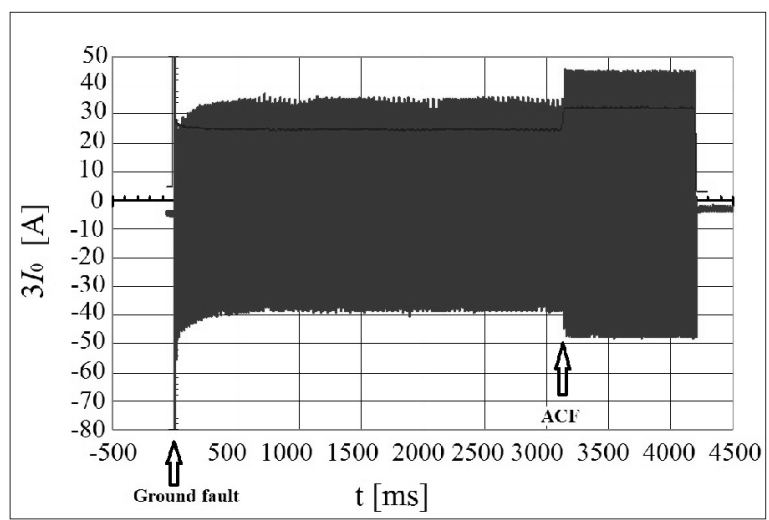

Fig. 14. Earth fault current $3 I_{0}$ in compensated network of $20 \mathrm{kV}$ with ACF system under permanent fault.

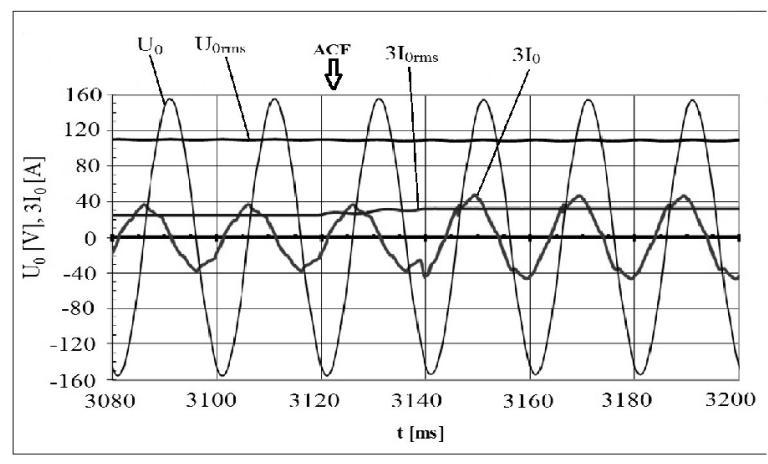

Fig. 15. A fragment of voltage $U_{0}$ and current $3 I_{0}$ waveforms in the compensated network of $20 \mathrm{kV}$ before and after the activation of the ACF system under permanent ground fault.

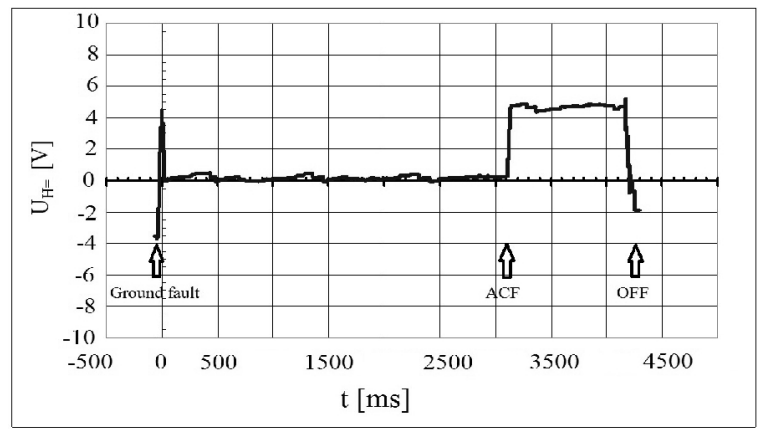

Fig. 16. Hall voltage DC component value before and after activation of the ACF in $20 \mathrm{kV}$ network under permanent ground fault.

For permanent ground faults in $20 \mathrm{kV}$ network with compensation and with an ACF system employed it can be stated that despite the relatively high value of a ground fault current (about 27 A - see Fig. 14), the output DC component of the Hall sensor is much below the operation threshold (around $0.5 \mathrm{~V}$ - see Fig. 16) due to the relay characteristic angle RCA equal to $0^{\circ}$. However, after activation of the ACF system (around $3 \mathrm{~s}$ ) the phase angle between $U_{0}$ and $3 I_{0}$ delivered to the Hall sensor is changed (see Fig. 15) and as a result the value of $U_{H}=$ reaches $4.5 \mathrm{~V}$ what improves effective clearing of the fault within approximately $1.5 \mathrm{~s}$ (see Fig. 16). (One can find that in this case after operation of the ACF system the ground fault current increased slightly to about $32 \mathrm{~A}$ ). Whereas, in a $20 \mathrm{kV}$ insulated network under permanent ground fault with low current values $(1.5 \mathrm{~A}-0.3 \mathrm{~A})$ the conventional ground fault protection was not able to detect these short-circuits. On the contrary the DC voltage value at the output of the
Hall sensor was sufficiently high to detect and to clear selectively such the ground faults what can be seen in Fig. 17.

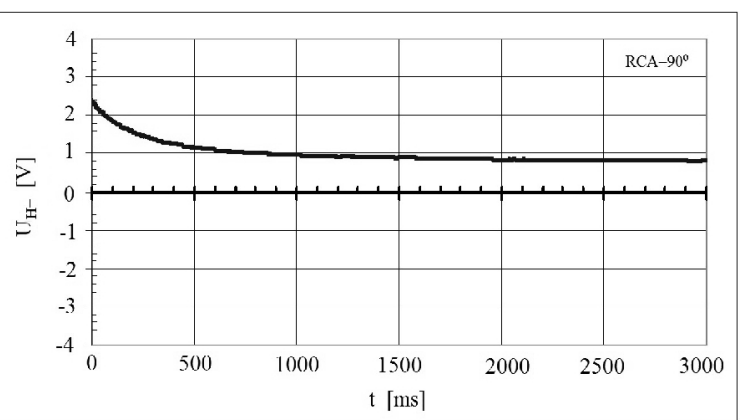

Fig. 17. The DC voltage component value of the Hall sensor during permanent ground fault in a $20 \mathrm{kV}$ network with insulated neutral point at low fault current value (1.5 A-0.3 A).

A similar situation occurs in $6 \mathrm{kV}$ networks as illustrated in Fig. 18-Fig. 20.

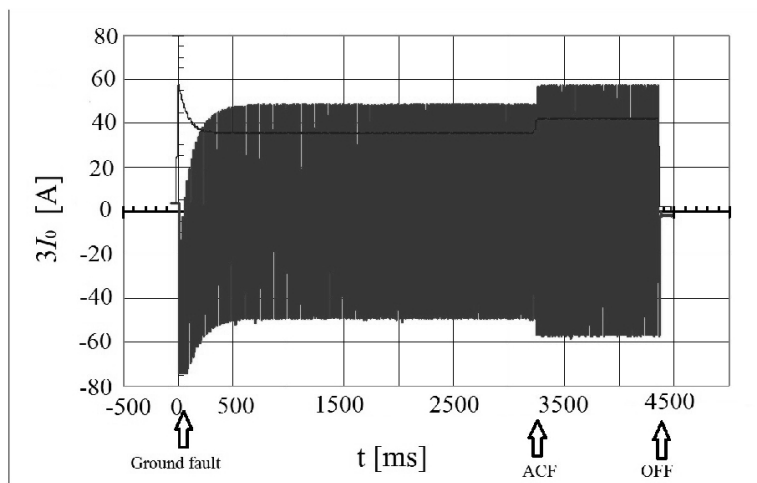

Fig. 18. Earth fault current $\left(3 I_{0}\right)$ in compensated $6 \mathrm{kV}$ network with ACF system under permanent fault.

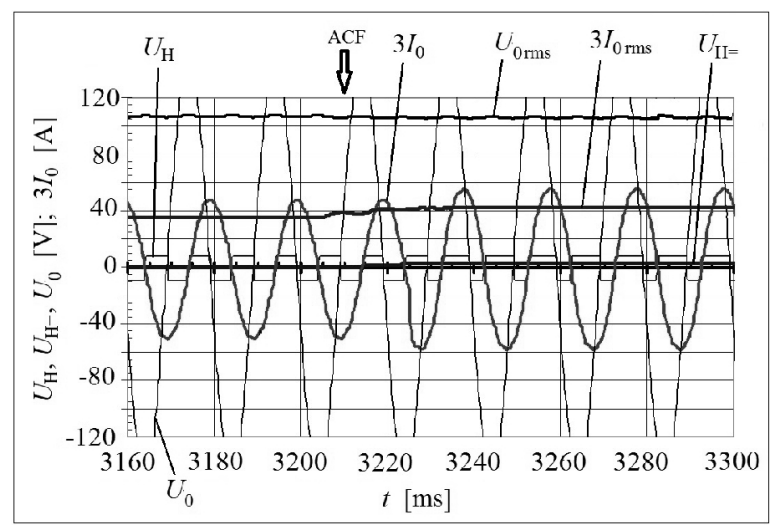

Fig. 19. The selected part of voltage $\left(U_{0}\right)$ and fault current $\left(3 I_{0}\right)$ waveform at the time of activation of the ACF system in compensated $6 \mathrm{kV}$ network under permanent ground fault.

The initial value of the short-circuit current was about $58 \mathrm{~A}$ and decreased next to about $37 \mathrm{~A}$. It increased respectively (to $48 \mathrm{~A}$ ) after the activation of the ACF system as illustrated in Fig. 18. The selected part of the $U_{0}$ voltage and fault current $\left(3 I_{0}\right)$ waveforms at the time of actuation of the ACF system together with variation of the Hall constant component $\left(U_{H=}\right)$ are presented in Fig. 19 and Fig. 20 respectively. Whereas, the value of the DC voltage Hall component of the protections installed on a healthy feeder is negative therefore, the protection is within the restrain zone (Fig. 21). 


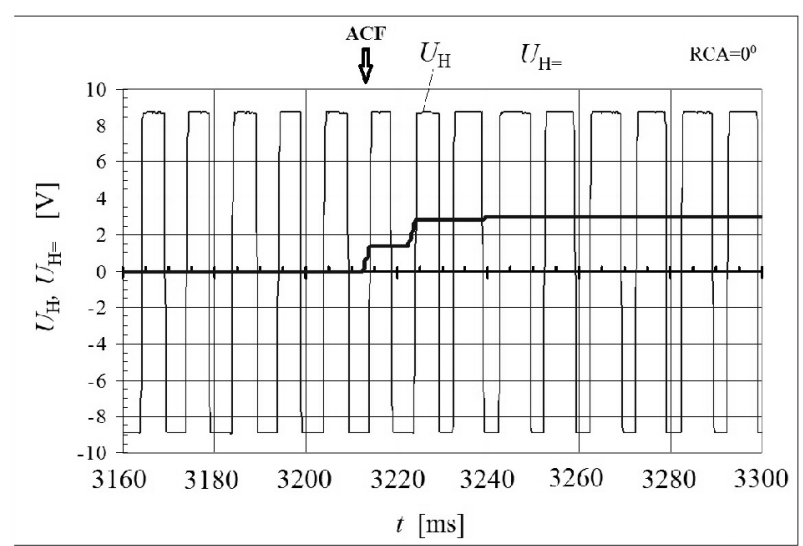

Fig. 20. Variation of variable $\left(U_{H}\right)$ and constant component $\left(U_{H=}\right)$ of the Hall sensor during permanent ground fault in compensated $6 \mathrm{kV}$ network with ACF system.

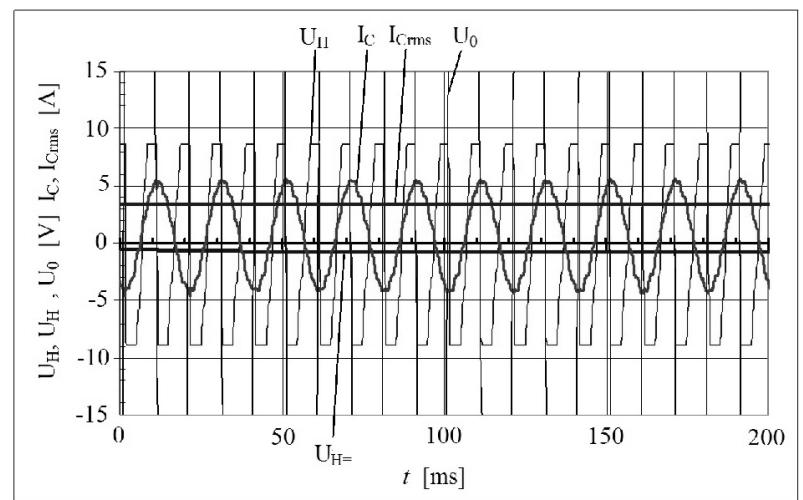

Fig. 21. Capacity leakage current $I_{c}$, zero-sequence voltage $U_{0}$ and Hall sensor output voltage $U_{H}$ during a permanent ground fault in a compensated $6 \mathrm{kV}$ network with the ACF system applied.

Quite important problem is to provide selective operation of the Hall based ground fault protection under significant load current of 3-phase cable and/or voltage unbalance conditions. It is particularly important during the start of induction motors directly from the network. In such cases one must use a suitable screening simultaneously with the establishment of respective threshold over expected residual voltage $U_{0}$ value [13], [15].

\section{CONCLUSIONS}

There is a real chance for effective use of a Hall sensor as the sensing element of directional earth fault protection in medium voltage power networks with no-effective earthing provided its proper selection for a new structure of a zerosequence balance current transformer.

The DC component of the Hall voltage as a result of product of zero sequence current and voltage affecting Hall sensor can be used as a criterion for protection operation.

To increase resistance of the protection to internal electromagnetic fields interference due to asymmetry of load as well as external interferences a special magnetic screen must be applied directly to the cable.

Investigations carried out under real ground faults of different types (solid, arcing and high resistance up to $2.5 \mathrm{k} \Omega$ ) in $6 \mathrm{kV}$ and $20 \mathrm{kV}$ distribution mine networks with no-effective earthing fully confirmed applicability of the newly developed directional ground fault protection with the Hall sensor as a discrimination unit. This protection is easy to use, maintained and managed.

\section{REFERENCES}

[1] Electricity Training Association, "Power System Protections," vol. 3, "Application", London, 1995.

[2] S. Hanninen, "Single phase earth faults in high impedance grounded networks. Characteristics, indication and location", Technical Research Centre of Finland, Espoo 2001. [Online]. Available: www.vtt.fi/inf/pdf/publications/2001/P453.pdf

[3] R. Mohammadi, H. A. Abyaneh, F. Razavi, et al., "Optimal relays coordination efficient method in interconnected power systems", Journal of Electrical Engineering-Elektrotechnicky Casopis, vol. 58, no. 5. pp. 271-278, 2007. [Online]. Available: iris.elf.stuba.sk/JEEEC/data/pdf/2110-02.pdf

[4] R. K. Panda, N. A. Sawade, "Air cored current sensor for digital metering \& micro-processor based protection unit", in Proc. of IEEE 5th Power India Conf., Murthal, pp. 1-6, 2012. [Online]. Available: http://dx.doi.org/10.1109/PowerI.2012.6479460

[5] Y. G. Zhang, Z. Zhao, Z. P. Wang, "Comprehensive detection and isolation of fault in complicated electrical engineering", Elektronika ir Elektrotechnika, no 9, pp. 31-34, 2013. [Online]. Available: $\mathrm{http}: / / \mathrm{dx}$.doi.org/10.5755/j01.eee.19.9.5646

[6] J. Izykowski, E. Rosolowski, P. Pierz, "Location of open conductor failure combined with phase-to-earth fault on power line", in Proc. 16th Int. Conf. Computational Problems of Electrical Engineering, (CPEE 2015), Lviv, Ukraine, 2015, pp. 56-58. [Online]. Available: http://dx.doi.org/10.1109/CPEE.2015.7333336

[7] M. Lukowicz, "New method of data transmission delay estimation for feeder differential protection", Present problems of power system control, vol. 1, pp. 92-103, 2011. [Online]. Available: www.psc.pwr.wroc.pl/files/PPoPSC 2011 Autumn.pdf

[8] N. I. Elkalashy, M. Lehtonen, H. A. Darwish, M. A. Izzularab, A. M. Taalab, "Modeling and experimental verification of high impedance arcing fault in medium voltage networks", IEEE Trans. on Dielectrics and Electrical Insulation, no. 2, pp. 375-383, 2007. [Online]. Available: http://dx.doi.org/10.1109/TDEI.2007.344617

[9] L. Marciniak, "Wavelet criteria for identification of arc intermittent faults in medium voltage networks", in Proc. IS Modern Electric Power Systems, (MEPS 2010), Wroclaw, Poland, 2010. [Online]. Available: http://ieeexplore.iee.org/xpl/articleDetails.jsp?ar number $=6479460$

[10] A. Sawicki, "Problems of modeling an electrical arc with variable geometric dimensions", Przeglad Elektrotechniczny, no. 2b, pp. 270275, 2013.

[11] W. Giriat, J. Rantuszkiewicz, "Hall sensors - Application of a Hall effect in techniques", $P W N$, Warszawa, 1961. (in Polish).

[12] Y-P. Tsai, K-L. Chen, Y-R. Chen, et al., "Multifunctional coreless hall-effect current transformer for the protection and measurement of power systems", IEEE Trans. on Instrumentation and Measurement, no. 3, pp. 557-565, 2014. [Online]. Available: http://dx.doi.org/10.1109/TIM.2013.2281555

[13] B. K. Wojszczyk, "Hall sensor in ground fault protections of MV network with controlled neutral point connection", Ph.D. dissertation, Wroclaw University of Technology, Poland, 2005.

[14] B. Miedzinski, W. Dzierzanowski, M. Habrych, X. Wang, Lj. Xu, "Analysis of magnetic field distribution in a Hall sensor based protection", Elektronika ir Elektrotechnika, no. 4, pp. 35-38, 2008, [Online]. Available: http://www.eejournal.ktu.lt/index.php/ elt/article/view/11121/5865

[15] B. Miedzinski, W. Dzierzanowski, M. Habrych, Z. Okraszewski, "The influence of magnetic shielding on selectivity of a Hall sensor based protection", Elektronika ir Elektrotechnika, no. 3, pp. 89-92, 2009. [Online]. Available: http://www.eejournal.ktu.lt/index.php/elt/article /view/10611/5408 